CORRECTION

https://doi.org/10.1038/s41586-019-0890-3

\title{
Author Correction: An experiment to search for dark-matter interactions using sodium iodide detectors
}

The COSINE-100 Collaboration

Correction to: Nature https://doi.org/10.1038/s41586-018-0739-1, published online 5 December 2018.

We wish to clarify that our results in this Letter do not have as broad a reach as originally indicated. Our data show that the annual modulation in the signal observed by DAMA is inconsistent with spin-independent interactions between weakly interacting massive particles (WIMPs) and sodium or iodine in the context of the standard halo model. Owing to an error during the production process, 'spin-independent' was omitted from a sentence in the Abstract, which should read: "Assuming the so-called standard dark-matter halo model, this result rules out spin-independent WIMP-nucleon interactions as the cause of the annual modulation observed by the DAMA collaboration". 